\title{
Multivalued Fixed Point Results for Two Families of Mappings in Modular-Like Metric Spaces with Applications
}

\author{
Tahair Rasham (D), ${ }^{1}$ Abdullah Shoaib $\mathbb{D}^{2},{ }^{2}$ Choonkil Park $\left(\mathbb{D},{ }^{3}\right.$ Manuel de la Sen $\mathbb{D}^{4}{ }^{4}$ \\ Hassen Aydi, ${ }^{5,6}$ and Jung Rye Lee ${ }^{7}$ \\ ${ }^{1}$ Department of Mathematics, Faculty of Science and Technology, Women University of Azad Jammu and Kashmir, \\ Bagh, Pakistan \\ ${ }^{2}$ Department of Mathematics and Statistics, Riphah International University, Islamabad, Pakistan \\ ${ }^{3}$ Research Institute for Natural Sciences, Hanyang University, Seoul 04763, Republic of Korea \\ ${ }^{4}$ Institute of Research and Development of Processes IIDP, University of the Basque Country, Campus of Leioa, P. O. Box 48940, \\ Leioa, 48940 Bizkaia, Spain \\ ${ }^{5}$ Université de Sousse, Institut Supérieur d'Informatique et des Techniques de Communication, H. Sousse 4000, Tunisia \\ ${ }^{6}$ China Medical University Hospital, China Medical University, Taichung 40402, Taiwan \\ ${ }^{7}$ Department of Mathematics, Daejin University, Kyunggi 11159, Republic of Korea
}

Correspondence should be addressed to Choonkil Park; baak@hanyang.ac.kr

Received 16 June 2020; Revised 7 August 2020; Accepted 23 October 2020; Published 16 November 2020

Academic Editor: Qingdu Li

Copyright ( $\odot 2020$ Tahair Rasham et al. This is an open access article distributed under the Creative Commons Attribution License, which permits unrestricted use, distribution, and reproduction in any medium, provided the original work is properly cited.

The aim of this research work is to find out some results in fixed point theory for a pair of families of multivalued mappings fulfilling a new type of $U$-contractions in modular-like metric spaces. Some new results in graph theory for multigraph-dominated contractions in modular-like metric spaces are developed. An application has been presented to ensure the uniqueness and existence of a solution of families of nonlinear integral equations.

\section{Introduction and Preliminaries}

If the image of a point $x$ under two mappings is $x$ itself, then $x$ is called a common fixed point of those mappings. Theory of fixed point has a basic role in analysis (see [1-39]). Chistyakov [7] established the concept of modular metric spaces and showed briefly about modular convergence, convex modular, equivalent metrics, abstract convex cone, and metric semigroup. Padcharoen et al. [16] introduced the concept of $\alpha$-type $F$-contractions in modular metric spaces and discussed some related results. Further results on these spaces in different directions can be seen in $[6,13,14]$.

In this paper, we establish some common fixed point theorems for two families of set-valued mappings satisfying a generalized contraction on a sequence only in a more generalized setting of modular-like metric spaces. New results can be established in dislocated metric spaces, ordered spaces, partial metric spaces, and metric spaces as a consequence of our findings. To support our results, some applications and examples are discussed. We give the following preliminary concepts which will be used in our results.

Definition 1. (see [16]). Let $A \neq \phi$. A function $w:(0, \infty) \times$ $A \times A \longrightarrow[0, \infty)$ is called a modular-like metric on $A$ if for all $a, b, c \in A, l>0$, and $w_{l}(a, b)=w(l, a, b)$, it satisfies

(i) $w_{l}(a, b)=w_{l}(b, a)$ for all $l>0$

(ii) $w_{l}(a, b)=0$ for all $l>0$ and then $a=b$

(iii) $w_{l+u}(a, b) \leq w_{l}(a, c)+w_{u}(c, b)$ for all $l, u>0$

If we replace (ii) by $w_{l}(a, b)=0$ for all $l>0$ if and only if $a=b$, then $w$ becomes a modular metric on $A$. If we replace (ii) by $w_{l}(a, b)=0$ for some $l>0$ and then $a=b$, then $w$ becomes a regular modular metric on $A$. For $g \in A$ and $\varepsilon>0$, $\overline{B_{w_{l}}(g, \varepsilon)}=\left\{p \in A: w_{l}(g, p) \leq \varepsilon\right\}$ is a closed ball in $(A, w)$. 
We will use m.l.m. space instead of modular-like metric space.

Definition 2. (see [16]). Let $(A, w)$ be an m.l.m. space.

(i) $E \subseteq A$ is known as $w$-complete if for any sequence $\left(a_{n}\right)_{n \in \mathbb{N}}$ in $E$ and for some $l>0, w_{l}\left(a_{m}, a_{n}\right) \longrightarrow 0$ as $m, n \longrightarrow \infty$ implies $w_{l}\left(a_{n}, a\right) \longrightarrow 0$ as $n \longrightarrow \infty$ for some $a \in E$

(ii) The sequence $\left(a_{n}\right)_{n \in \mathbb{N}}$ in $A$ is known as $w$-Cauchy for some $l>0$ if $w_{l}\left(a_{m}, a_{n}\right) \longrightarrow 0$ as $m, n \longrightarrow \infty$

(iii) The sequence $\left(a_{n}\right)_{n \in \mathbb{N}}$ in $A$ is known as $w$-convergent to $a \in A$ for some $l>0$ if and only if $w_{l}\left(a_{n}, a\right) \longrightarrow 0$ as $n \longrightarrow \infty$

Definition 3. Let $(A, w)$ be an m.l.m. space and $E \subseteq A$. A member $p_{0}$ which belongs to $E$ is said to be a best approximation in $E$ for $g \in A$ if

$$
w_{l}(g, E)=\inf _{p \in E} w_{l}(g, p)=w_{l}\left(g, p_{0}\right) .
$$

If each $g \in A$ has a best approximation in $E$, then $E$ is known as a proximinal set. $P(A)$ is equal to the family of proximinal sets in $A$. Let $A=\mathbb{R}^{+} \cup\{0\}$ and $w_{l}(g, p)=1 / l(g+p)$ for all $l>0$. Define a set $E=[4,6]$; then, for each $y \in A$,

$$
w_{l}(y, E)=w_{l}(y,[4,6])=\inf _{u \in[4,6]} w_{l}(y, u)=w_{l}(y, 4) .
$$

Hence, 4 is the best approximation in $E$ for each $y \in A$. Also, $[4,6]$ is a proximinal set.

Definition 4. The set-valued mapping $H_{w_{l}}: P(A) \times P(A)$ $\longrightarrow[0, \infty)$, defined by

$$
H_{w_{l}}(N, M)=\max \left\{\sup _{n \in N} w_{l}(n, M), \sup _{m \in M} w_{l}(N, m)\right\},
$$

is known as $w_{l}$-Hausdorff metric. The pair $\left(P(A), H_{w_{l}}\right)$ is called the $w_{l}$-Hausdorff metric space. Let $A=\mathbb{R}^{+} \cup\{0\}$ and $w_{l}(g, p)=(1 / l)(g+p)$ for all $l>0$. If $P=[5,6]$ and $O=[9,10]$, then $H_{w_{l}}(P, O)=(13 / l)$.

Definition 5. Let $(X, w)$ be a modular-like metric space. Then, we will say that $w$ satisfies the $\Delta_{M}$-condition if it is the case that $\lim _{n, m \rightarrow \infty} w_{p}\left(x_{n}, x_{m}\right)=0$, for $p=m-n$ implies $\lim _{n, m \longrightarrow \infty} w_{l}\left(x_{n}, x_{m}\right)=0(m, n \in \mathbb{N}, m>n)$ for some $l>0$.

Definition 6 (see [33]). Let $A \neq \phi, \xi: A \longrightarrow P(A)$ be a setvalued mapping, $B \subseteq A$, and $\alpha: A \times A \longrightarrow[0,+\infty)$. Then, $\xi$ is called $\alpha_{*}$-admissible on $K$ if $\alpha_{*}(\xi a, \xi c)=\inf \{\alpha(u, v): u \in \xi a, v \in \xi c\} \geq 1 \quad$ whenever $\alpha(a, c) \geq 1$, for all $a, c \in B$.

Definition 7. Let $A \neq \phi, \xi: A \longrightarrow P(A)$ be a set-valued mapping, $M \subseteq A$, and $\alpha: A \times A \longrightarrow[0,+\infty)$. Then, $\xi$ is called $\alpha_{*}$-dominated on $M$ if for all $a \in M, \alpha_{*}(a, \xi a)=\inf \{\alpha(a, l): l \in \xi a\} \geq 1$.
Definition 8. (see [39]). Consider a metric space $(W, d)$. A function $H: W \longrightarrow W$ is said $A$-contraction if for all $c, k \in W$, there exists $\tau>0$ such that $d(H a, H c)>0$ implies

$$
\tau+A(d(H a, H c)) \leq A(d(a, c)),
$$

where $A: \mathbb{R}_{+} \longrightarrow \mathbb{R}$ is a mapping which satisfies the following:

(F1) There exists $k \in(0,1)$ such that $\lim _{\sigma \longrightarrow 0^{+}} \sigma^{k} A(\sigma)=0$

(F2) For all $a, c \in \mathbb{R}_{+}$such that $a<c, A(a)<A(c)$, that is, $A$ is strictly increasing

(F3) $\quad \lim _{n \longrightarrow+\infty} \sigma_{n}=0$ if and only if $\lim _{n \longrightarrow+\infty} A\left(\sigma_{n}\right)=-\infty$, for each sequence $\left\{\sigma_{n}\right\}_{n=1}^{\infty}$ of positive numbers

The family of all mappings satisfying conditions (F1) to (F3) is denoted by F.

Lemma 1. Let $(A, w)$ be an m.l.m. space. Let $\left(P(A), H_{w_{l}}\right)$ be a Hausdorff $w_{l}$-metric-like space. Then, for each $a \in K$ and for all $K, M \in P(A)$, there exists $b_{a} \in M$ such that $H_{w_{l}}(K, M) \geq w_{l}\left(a, b_{a}\right)$.

Proof. If $\quad H_{w_{l}}(K, M)=\sup _{a \in K} w_{l}(a, M), \quad$ then $H_{w_{l}}(K, M) \geq w_{l}(a, M)$ for each $a \in K$. As $M$ is the proximinal set, for every $a \in A$, there exists at least one best approximation $b_{a} \in M$ which satisfies $w_{l}(a, M)=w_{l}\left(a, b_{a}\right)$. Now, we have $H_{w_{l}}(K, M) \geq w_{l}\left(a, b_{a}\right)$. Now,

$$
\begin{aligned}
H_{w_{l}}(K, M)= & \sup _{h \in M} w_{l}(K, h) \geq \sup _{a \in K} w_{l}(a, M) \geq w_{l}\left(a, b_{a}\right), \\
& \text { for some } b_{a} \in M .
\end{aligned}
$$

Hence proved.

Example 1. (see [21]). Let $A=\mathbb{R}$. Define $B: A \times A \longrightarrow[0, \infty)$ by

$$
B(b, t)= \begin{cases}1 & \text { if } b>t, \\ \frac{1}{4} & \text { if } b \ngtr t .\end{cases}
$$

Define $G, M: A \longrightarrow P(A)$ by

$$
\begin{aligned}
G b & =[-4+b,-3+b], \\
M t & =[-2+t,-1+t] .
\end{aligned}
$$

Then, $G$ and $M$ are not $\alpha_{*}$-admissible, but they are $\alpha_{*}$-dominated.

\section{Main Results}

Let $(\mathfrak{I}, w)$ be an m.l.m. space and $c_{0} \in \mathfrak{J}$; let $\left\{S_{\sigma}: \sigma \in \Omega\right\}$ and $\left\{T_{\beta}: \beta \in \Phi\right\}$ be two families of multifunctions from $\mathfrak{I}$ to $P(\Im)$ Let $c_{1} \in S_{a} c_{0}$ be an element such that $w\left(c_{0}, S_{a} c_{0}\right)=w\left(c_{0}, c_{1}\right)$. Let $c_{2} \in T_{b} c_{1}$ be such that $w\left(c_{1}, T_{b} c_{1}\right)=w\left(c_{1}, c_{2}\right)$. Let $c_{3} \in S_{c} c_{2}$ such that 
$w\left(c_{2}, S_{c} c_{2}\right)=w\left(c_{2}, c_{3}\right)$. In this way, we get a sequence $\left\{T_{\beta} S_{\sigma}\left(c_{n}\right)\right\}$ in $\mathfrak{J}$, where $c_{2 n+1} \in S_{i} c_{2 n}, c_{2 n+2} \in T_{j} c_{2 n+1}, n \in \mathbb{N}$, $i \in \Omega$, and $j \in \Phi$. Also, $w\left(c_{2 n}, S_{i} c_{2 n}\right)=w\left(c_{2 n}, c_{2 n+1}\right)$ and $w\left(c_{2 n+1}, T_{j} c_{2 n+1}\right)=w\left(c_{2 n+1}, c_{2 n+2}\right) .\left\{T_{\beta} S_{\sigma}\left(c_{n}\right)\right\}$ is said to be a sequence in $\Im$ generated by $c_{0}$. If $\left\{S_{\sigma}: \sigma \in \Omega\right\}=\left\{T_{\beta}: \beta \in \Phi\right\}$, then we denote $\left\{S_{\sigma}\left(c_{n}\right)\right\}$ instead of $\left\{T_{\beta} S_{\sigma}\left(c_{n}\right)\right\}$.
Theorem 1. Let $(\mathfrak{I}, w)$ be a complete m.l.m. space. Assume that $w$ is regular and satisfies the $\Delta_{M}$-condition. Let $c_{0} \in \mathfrak{I}$, $\alpha: \mathfrak{I} \times \mathfrak{J} \longrightarrow[0, \infty),\left\{T_{\beta}: \beta \in \Phi\right\}$, and $\left\{S_{\sigma}: \sigma \in \Omega\right\}$ be the families of $\alpha_{*}$-dominated set-valued functions on $w$. Suppose there exist $\tau>0$ and $U \in \mathrm{F}$ such that

$$
\tau+U\left(H_{w_{1}}\left(S_{\sigma} t, T_{\beta} g\right)\right) \leq U\left(\max \left\{w_{1}(t, g), w_{1}\left(t, S_{\sigma} t\right), \frac{w_{2}\left(t, T_{\beta} g\right)}{2}, \frac{w_{1}\left(t, S_{\sigma} t\right) \cdot w_{1}\left(g, T_{\beta} g\right)}{1+w_{1}(t, g)}\right\}\right)
$$

whenever $t, g \in \mathfrak{J} \cap\left\{T_{\beta} S_{\sigma}\left(c_{n}\right)\right\}, \quad \alpha(t, g) \geq 1, \sigma \in \Omega, \beta \in \Phi$, and $H_{w_{1}}\left(S_{\sigma} t, T_{\beta} g\right)>0$.

Then, the sequence $\left\{T_{\beta} S_{\sigma}\left(c_{n}\right)\right\}$ generated by $c_{0}$ converges to $c \in \mathfrak{J}$, and for each $n \in \mathbb{N}, \alpha\left(c_{n}, c_{n+1}\right) \geq 1$. Also, if $c$ satisfies (8) and either $\alpha\left(c_{n}, c\right) \geq 1$ or $\alpha\left(c, c_{n}\right) \geq 1$ for all $n \in \mathbb{N} \cup\{0\}$, then $S_{\sigma}$ and $T_{\beta}$ have a common fixed point $c$ in $\mathfrak{\Im}$ for all $\sigma \in \Omega$ and $\beta \in \Phi$.
Proof. Consider a sequence $\left\{T_{\beta} S_{\sigma}\left(c_{n}\right)\right\}$. Obviously, $c_{n} \in \mathfrak{I}$ for each $n \in \mathbb{N}$. If $j$ is odd, then $j=2+1$ for some $\in \mathbb{N}$. By the definition of $\alpha_{*}$-dominated mappings, we have $\alpha_{*}\left(c_{2^{\prime}}, S_{\sigma} c_{2^{\prime}}\right) \geq 1$ and $\alpha_{*}\left(c_{2^{*}+1}, T_{\beta} c_{2^{*}+1}\right) \geq 1$ for all $\sigma \in \Omega$ and $\beta \in \Phi$. As $\quad \alpha_{*}\left(c_{2}, S_{\sigma} c_{2}\right) \geq 1$, this implies $\inf \left\{\alpha\left(c_{2}, b\right): b \in S_{\sigma} c_{2}\right\} \geq 1$. Also, $c_{2^{2}+1} \in S_{f} c_{2}$ for some $f \in \Omega$, so $\alpha\left(c_{2^{\prime}}, c_{2^{\prime}+1}\right) \geq 1$. Also, $c_{2^{+}+2} \in T_{g} c_{2^{\prime}+1}$ for some $g \in \Phi$. Now, by using Lemma 1 , we have

$$
\begin{aligned}
& \tau+U\left(w_{1}\left(c_{2^{\prime}+1}, c_{2^{\prime}+2}\right)\right) \leq \tau+U\left(H_{w_{1}}\left(S_{f} c_{2^{\prime}}, T_{g} c_{2^{\prime}+1}\right)\right) \\
& \leq U\left(\max \left\{w_{1}\left(c_{2^{\prime}}, c_{2^{\prime}+1}\right), w_{1}\left(c_{2^{\prime}}, S_{f} c_{2^{\prime}}\right), \frac{w_{2}\left(c_{2^{\prime}}, T_{g} c_{2^{\prime}+1}\right)}{2}, \frac{w_{1}\left(c_{2^{\prime}}, S_{f} c_{2^{\prime}}\right) \cdot w_{1}\left(c_{2^{\prime}+1}, T_{g} c_{2^{\prime}+1}\right)}{1+w_{1}\left(c_{2^{\prime}}, c_{2^{\prime}+1}\right)}\right\}\right) \\
& \leq U\left(\max \left\{w_{1}\left(c_{2^{\prime}}, c_{2^{\prime}+1}\right), w_{1}\left(c_{2^{\prime}}, c_{2^{\prime}+1}\right), \frac{w_{1}\left(c_{2^{\prime}}, c_{2^{\prime}+1}\right)+w_{1}\left(c_{2^{\prime}+1}, c_{2^{\prime}+2}\right)}{2}, \frac{w_{1}\left(c_{2^{\prime}}, c_{2^{\prime}+1}\right) \cdot w_{1}\left(c_{2^{\prime}+1}, c_{2^{\prime}+2}\right)}{1+w_{1}\left(c_{2^{\prime}}, c_{2^{\prime}+1}\right)}\right\}\right) \\
& \leq U\left(\max \left\{w_{1}\left(c_{2^{\prime}}, c_{2^{\prime}+1}\right), w_{1}\left(c_{2^{\prime}+1}, c_{2^{\prime}+2}\right)\right\}\right) .
\end{aligned}
$$

This implies

$\tau+U\left(w_{1}\left(c_{2^{2}+1}, c_{2^{4}+2}\right)\right) \leq U\left(\max \left\{w_{1}\left(c_{2^{2}}, c_{2^{4}+1}\right), w_{1}\left(c_{2^{4}+1}, c_{2^{2}+2}\right)\right\}\right)$.

Now, if

$$
\max \left\{w_{1}\left(c_{2^{\prime}}, c_{2^{\prime}+1}\right), w_{1}\left(c_{2^{\prime}+1}, c_{2^{\prime}+2}\right)\right\}=w_{1}\left(c_{2^{\prime}+1}, c_{2^{\prime}+2}\right),
$$

then from (10), we have

$$
U\left(w_{1}\left(c_{2 i+1}, c_{2 i+2}\right)\right) \leq U\left(w_{1}\left(c_{2 i+1}, c_{2 i+2}\right)\right)-\tau,
$$

a contradiction. Therefore,

$$
\max \left\{w_{1}\left(c_{2^{\prime}}, c_{2^{\prime}+1}\right), w_{1}\left(c_{2^{\prime}+1}, c_{2^{\prime}+2}\right)\right\}=w_{1}\left(c_{2^{\prime}}, c_{2^{\prime}+1}\right),
$$

for all $i \in \mathbb{N} \cup\{0\}$. Hence, from (10), we have

$$
U\left(w_{1}\left(c_{2 i+1}, c_{2 i+2}\right)\right) \leq U\left(w_{1}\left(c_{2 i}, c_{2 i+1}\right)\right)-\tau .
$$

Similarly, we have

$$
U\left(w_{1}\left(c_{2 i}, c_{2 i+1}\right)\right) \leq U\left(w_{1}\left(c_{2 i-1}, c_{2 i}\right)\right)-\tau,
$$

for all $i \in \mathbb{N} \cup\{0\}$. By (14) and (15), we have

$$
U\left(w_{1}\left(c_{2 i+1}, c_{2 i+2}\right)\right) \leq U\left(w_{1}\left(c_{2 i-1}, c_{2 i}\right)\right)-2 \tau .
$$

Repeating these steps, we get

$$
U\left(w_{1}\left(c_{2 i+1}, c_{2 i+2}\right)\right) \leq U\left(w_{1}\left(c_{0}, c_{1}\right)\right)-(2 i+1) \tau .
$$

Similarly, we have

$$
U\left(w_{1}\left(c_{2 i}, c_{2 i+1}\right)\right) \leq U\left(w_{1}\left(c_{0}, c_{1}\right)\right)-2 i \tau .
$$

Inequalities (17) and (18) can jointly be written as

$$
U\left(w_{1}\left(c_{n}, c_{n+1}\right)\right) \leq U\left(w_{1}\left(c_{0}, c_{1}\right)\right)-n \tau .
$$

Taking the limit as $n \longrightarrow \infty$ in (19), we have

$$
\lim _{n \longrightarrow \infty} U\left(w_{1}\left(c_{n}, c_{n+1}\right)\right)=-\infty .
$$

Since $U \in \mathrm{F}$,

$$
\lim _{n \longrightarrow \infty} w_{1}\left(c_{n}, c_{n+1}\right)=0 .
$$

Applying the property (F1) of $\mathrm{F}$, there exists $k \in(0,1)$ such that 


$$
\lim _{n \longrightarrow \infty}\left(w_{1}\left(c_{n}, c_{n+1}\right)\right)^{k}\left(U\left(w_{1}\left(c_{n}, c_{n+1}\right)\right)\right)=0 .
$$

By (19), for all $n \in \mathbb{N}$, we obtain

$$
\left(w_{1}\left(c_{n}, c_{n+1}\right)\right)^{k}\left(U\left(w_{1}\left(c_{n}, c_{n+1}\right)\right)\right)-U\left(w_{1}\left(c_{0}, c_{1}\right)\right) \leq-\left(w_{1}\left(c_{n}, c_{n+1}\right)\right)^{k} n \tau \leq 0 .
$$

Considering (21) and (22) and letting $n \longrightarrow \infty$ in (23), we have

$$
\lim _{n \rightarrow \infty} n\left(w_{1}\left(c_{n}, c_{n+1}\right)\right)^{k}=0 .
$$

$$
w_{1}\left(c_{n}, c_{n+1}\right) \leq \frac{1}{n^{(1 / k)}} \quad \text { for all } n \geq n_{1} .
$$

Take $p>0$ and $m=n+p>n>n_{1}$; then,

Since (24) holds, there exists $n_{1} \in \mathbb{N}$ such that $n\left(w_{1}\left(c_{n}, c_{n+1}\right)\right)^{k} \leq 1$ for all $n \geq n_{1}$ or

$$
w_{p}\left(c_{n}, c_{m}\right) \leq w_{1}\left(c_{n}, c_{n+1}\right)+w_{1}\left(c_{n+1}, c_{n+2}\right)+\cdots+w_{1}\left(c_{m}, c_{m+1}\right) \leq \frac{1}{n^{(1 / k)}}+\frac{1}{(n+1)^{(1 / k)}}+\cdots+\frac{1}{m^{(1 / k)}} .
$$

Applying the limit as $n, m \longrightarrow \infty$ on both sides, we have

$$
\lim _{n, m \longrightarrow \infty} w_{p}\left(c_{n}, c_{m}\right) \leq \lim _{n, m \longrightarrow \infty} \frac{1}{n^{(1 / k)}}+\lim _{n, m \longrightarrow \infty} \frac{1}{(n+1)^{(1 / k)}}+\cdots+\lim _{n, m \longrightarrow \infty} \frac{1}{m^{(1 / k)}}
$$

As $k \in(0,1)$, then $(1 / k)>1$, and so,

$\lim _{n, m \longrightarrow \infty} \frac{1}{n^{(1 / k)}}=\lim _{n, m \longrightarrow \infty} \frac{1}{(n+1)^{(1 / k)}}=\lim _{n, m \longrightarrow \infty} \frac{1}{m^{(1 / k)}}=0$.

As $w:(0, \infty) \times \mathfrak{I} \times \mathfrak{I} \longrightarrow[0, \infty)$, then

$$
\lim _{n, m \rightarrow \infty} w_{p}\left(c_{n}, c_{m}\right)=0 \text {. }
$$

Since $w$ satisfies the $\Delta_{M}$-condition, we have

$$
\lim _{n, m \rightarrow \infty} w_{1}\left(c_{n}, c_{m}\right)=0 .
$$

Hence, $\left\{T_{\beta} S_{\sigma}\left(c_{n}\right)\right\}$ is a Cauchy sequence in $w$. Since $(\mathfrak{J}, w)$ is a regular complete modular-like metric space, there exists $c \in \mathfrak{J}$ such that $\left\{T_{\beta} S_{\sigma}\left(c_{n}\right)\right\} \longrightarrow c$ as $n \longrightarrow \infty$, and so,

$$
\lim _{n \longrightarrow \infty} w_{1}\left(c_{n}, c\right)=0
$$

Now, by Lemma 1, we have

$$
\tau+U\left(w_{1}\left(c_{2 n+1}, T_{\beta} c\right)\right) \leq \tau+U\left(H_{w_{1}}\left(S_{e} c_{2 n}, T_{\beta}{ }^{\prime} c\right)\right),
$$

for some $\beta \in \Phi$ and some $e \in \Omega$. Now, there exists $c_{2 n+1} \in S_{e} c_{2 n}$ such that $w_{1}\left(c_{2 n}, S_{e} c_{2 n}\right)=w_{1}\left(c_{2 n}, c_{2 n+1}\right)$. From the assumption, $\alpha\left(c_{n}, c\right) \geq 1$. Assume that $w_{1}\left(c, T_{\beta} c\right)>0$; then, there must be a positive real number $p$, such that $w_{1}\left(c_{2 n+1}, T_{\beta} c\right)>0$ for $n \geq p$. Now, $H_{w_{1}}\left(S_{e} c_{2 n}, T_{\beta} c\right)>0$; then, by using inequality (8), we have

$$
\tau+U\left(w_{1}\left(c_{2 n+1}, T_{\beta} c^{\prime}\right)\right) \leq U\left(\max \left\{w_{1}\left(c_{2 n}, \dot{c}\right), w_{1}\left(c_{2 n}, c\right), \frac{w_{1}\left(c_{2 n}, c_{2 n+1}\right)+w_{1}\left(c_{2 n+1}, T_{\beta} c\right)}{2}, \frac{w_{1}\left(c_{2 n}, S_{e} c_{2 n}\right) \cdot w_{1}\left(f, T_{\beta} c\right)}{1+w_{1}\left(c_{2 n}, c\right)}\right\}\right)
$$

Letting $n \longrightarrow \infty$ and using (31), we get

$$
\tau+U\left(w_{1}\left(\dot{c}, T_{\beta}^{\prime} c\right)\right) \leq U\left(w_{1}\left(\dot{c}, T_{\beta}^{\prime} c\right)\right)
$$

Since $U$ is strictly increasing, (32) implies

$$
w_{1}\left(\dot{c}, T_{\beta} c^{\prime}\right)<w_{1}\left(\dot{c}, T_{\beta}{ }^{\prime}\right)
$$

This is not true, So, our assumption is wrong. Hence, $w_{1}\left(c, T_{\beta} c\right)=0$ or $c \in T_{\beta} c$ for each $\beta \in \Phi$. Similarly, by proceeding with Lemma 1 and inequality (8), we can prove that $w_{1}\left(c, S_{\sigma} c\right)=0$ or $c \in S_{\sigma} c$ for all $\sigma \in \Omega$. Hence, $c$ is a 
common fixed point of both the mappings $S_{\sigma}$ and $T_{\beta}$ in $\mathfrak{\Im}$ for all $\sigma \in \Omega$ and $\beta \in \Phi$.

Example 2. Let $\mathfrak{I}=\mathbb{R}_{+} \cup\{0\}$. Take $w_{2}(q, g)=(q+g)$ and $w_{1}(q, g)=1 / 2(q+g)$ for all $q, g \in \mathfrak{J}$. Suppose that $S_{\sigma}, T_{\beta}: \mathfrak{I} \times \mathfrak{I} \longrightarrow P(\mathfrak{I})$ are two families of multivalued mappings defined by

$$
S_{m} v=\left[\frac{v}{3 m}, \frac{2 v}{3 m}\right] \text { if } v \in \mathfrak{I},
$$

where $m=1,2,3, \ldots$, and

$$
T_{n} v=\left[\frac{v}{4 n}, \frac{3 v}{4 n}\right] \quad \text { if } v \in \mathfrak{I}
$$

where $n=1,2,3, \ldots$. Suppose that $v_{0}=1$ and $w_{1}\left(v_{0}\right.$, $\left.S_{1} v_{0}\right)=w_{1}\left(1, S_{1} 1\right)=w_{1}(1,(1 / 3))$. So, $v_{1}=(1 / 3)$. Now, $w_{1}\left(v_{1}, T_{1} v_{1}\right)=w_{1}\left((1 / 3), T_{1}(1 / 3)\right)=w_{1}((1 / 3),(1 / 12))$. So, $v_{2}=(1 / 12)$. Now, $w_{1}\left(v_{2}, S_{2} v_{2}\right)=w_{1}\left((1 / 12), S_{2}(1 / 12)\right)=$ $w_{1}((1 / 12),(1 / 72))$. So, $v_{3}=(1 / 72)$. Continuing in this way, we have $\left\{T_{n} S_{m}\left(v_{n}\right)\right\}=\{1,(1 / 3),(1 / 12),(1 / 72), \ldots\}$. Consider the mapping $\alpha: \mathfrak{I} \times \mathfrak{I} \longrightarrow[0, \infty)$ defined by

$$
\alpha(r, t)= \begin{cases}1, & \text { if } r>t, \\ \frac{1}{2}, & \text { otherwise. }\end{cases}
$$

Now, if $v, y \in \mathfrak{I} \cap\left\{T_{\beta} S_{\sigma}\left(v_{n}\right)\right\}$ with $\alpha(v, y) \geq 1$, we have

$$
\begin{aligned}
& H_{w_{1}}\left(S_{m} v, T_{n} y\right)=\max \left\{\sup _{a \in S_{m} x} w_{1}\left(a, T_{n} y\right), \sup _{b \in T_{n} y} w_{1}\left(S_{m} v, b\right)\right\} \\
& =\max \left\{\begin{array}{c}
w_{1}\left(\frac{2 v}{3 m},\left[\frac{y}{4 n}, \frac{3 y}{4 n}\right]\right), \\
w_{1}\left(\left[\frac{v}{3 m}, \frac{2 v}{3 m}\right], \frac{3 y}{4 n}\right)
\end{array}\right\} \\
& =\max \left\{w_{1}\left(\frac{2 v}{3 m}, \frac{y}{4 n}\right), w_{1}\left(\frac{v}{3 m}, \frac{3 y}{4 n}\right)\right\}=\frac{1}{2} \max \left\{\frac{2 v}{3 m}+\frac{y}{4 n}, \frac{v}{3 m}+\frac{3 y}{4 n}\right\}, \\
& W(x, y)=\max \left(\frac{1}{2}\left\{\begin{array}{c}
v+y, v+\frac{v}{3 m}, \\
v+\frac{y}{4 n}, \frac{(v+(v / 3 m)) \cdot(y+(y / 4 n))}{\{1+v+y\}}
\end{array}\right\}\right) .
\end{aligned}
$$

Case (i). If $\max \{(2 v / 3 m)+(y / 4 n),(y / 3 m)+(3 y /$

$4 n)\}=(2 v / 3 m)+(y / 4 n)$ and $\tau=\ln (1.2)$, then we have

$$
\begin{aligned}
& 8 v n+3 m y \leq 10 m n x+10 m n y, \\
& 48 v n+18 m y \leq 60 m n x+60 m n y, \\
& \frac{6}{5}\left(\frac{2 v}{3 m}+\frac{y}{4 n}\right) \leq x+y, \\
& \ln (1.2)+\ln \left(\frac{2 v}{3 m}+\frac{y}{4 n}\right) \leq \ln (x+y) .
\end{aligned}
$$

This implies that

$$
\tau+U\left(H_{w_{1}}\left(S_{m} v, T_{n} y\right)\right) \leq U(W(x, y)) .
$$

Case (ii). If $\max \{2 v / 3 m+y / 4 n, y / 3 m+3 y / 4 n\}=v / 3 m+$ $3 y / 4 n$ and $\tau=\ln (1.2)$, then we have

$$
4 v n+9 m y \leq 10 m n x+10 m n y,
$$$$
24 v n+54 m y \leq 60 m n x+60 m n y,
$$$$
\frac{6}{5}\left(\frac{v}{3 m}+\frac{3 y}{4 n}\right) \leq x+y
$$

$$
\ln (1.2)+\ln \left(\frac{v}{3 m}+\frac{3 y}{4 n}\right) \leq \ln (x+y) .
$$

This implies that

$$
\tau+U\left(H_{w_{1}}\left(S_{m} v, T_{n} y\right)\right) \leq U(W(x, y)) .
$$

Hence, all the requirements of Theorem 1 are satisfied. So, the families $\left\{S_{m}\right\}$ and $\left\{T_{n}\right\}$ have a common fixed point.

If we take $\left\{S_{\sigma}: \sigma \in \Omega\right\}=\left\{T_{\beta}: \beta \in \Phi\right\}$ in Theorem 1, then we obtain the above result.

Corollary 1. Let $(\mathfrak{\Im}, w)$ be a complete m.l.m. space. Assume that $w$ is regular and satisfies the $\Delta_{M}$-condition. Let $c_{0} \in \mathfrak{I}$, $\alpha: \mathfrak{J} \times \mathfrak{J} \longrightarrow[0, \infty)$, and $\left\{S_{\sigma}: \sigma \in \Omega\right\}$ be a family of 
$\alpha_{*}$-dominated set-valued functions on $\mathfrak{\Im}$. If there exist $\tau>0$ and $U \in \mathrm{F}$ such that

$$
\tau+U\left(H_{w_{l}}\left(S_{\sigma} t, S_{\beta} g\right)\right) \leq U\left(\max \left\{w_{1}(t, g), w_{1}\left(t, S_{\sigma} t\right), w_{2}\left(t, S_{\beta} g\right), \frac{w_{1}\left(t, S_{\sigma} t\right) \cdot w_{1}\left(g, S_{\beta} g\right)}{1+w_{1}(t, g)}\right\}\right)
$$

whenever $t, g \in \mathfrak{I} \cap\left\{S_{\sigma}\left(c_{n}\right)\right\}, \quad \alpha(t, g) \geq 1, \quad \sigma, \beta \in \Omega$, and $H_{w_{1}}\left(S_{\sigma} t, S_{\beta} g\right)>0$, then the sequence $\left\{\mathfrak{J} S_{\sigma}\left(c_{n}\right)\right\}$ generated by $c_{0}$ converges to $c \in \mathfrak{J}$, and for each $n \in \mathbb{N}, \alpha\left(c_{n}, c_{n+1}\right) \geq 1$. Also, if $c$ satisfies (44) and either $\alpha\left(c_{n}, c\right) \geq 1$ or $\alpha\left(c, c_{n}\right) \geq 1$ for every $n \in \mathbb{N} \cup\{0\}$, then $\left\{S_{\sigma}: \sigma \in \Omega\right\}$ has a common fixed point $c$ in $\mathfrak{\Im}$.

\section{Applications in Graph Theory}

Jachymski [11] developed a relation between fixed point theory and graph theory by the induction of graphic contractions. Hussain et al. [9] established some results for the new type of contractions endowed with a graph and also showed an application. Further useful results on the graph can be seen in $[34,35,40]$.
Definition 9. Let $A$ be a nonempty set and $Y=(V(Y), L(Y))$ be a graph with $V(Y)=A$. A mapping $F$ from $A$ to $P(A)$ is known as multigraph-dominated on $A$ if $(a, b) \in L(Y)$, whenever $a \in A$ and $b \in F a$.

Theorem 2. Let $(\mathfrak{I}, w)$ be a complete m.l.m. space endowed with a graph $Y, c_{0} \in \mathfrak{I}$, and the following hold:
(i) $\left\{T_{\beta}: \beta \in \Phi\right\}$ and $\left\{S_{\sigma}: \sigma \in \Omega\right\}$ are sets of multigraph- dominated functions on $\mathfrak{\Im} \cap\left\{T_{\beta} S_{\sigma}\left(c_{n}\right)\right\}$.

(ii) There exist $\tau>0$ and $U \in F$ such that

$$
\tau+U\left(H_{w_{1}}\left(S_{\sigma} t, T_{\beta} y\right)\right) \leq U\left(\max \left\{w_{1}(t, y), w_{1}\left(t, S_{\sigma} t\right), \frac{w_{2}\left(t, T_{\beta} y\right)}{2}, \frac{w_{1}\left(t, S_{\sigma} t\right) \cdot w_{1}\left(y, T_{\beta} y\right)}{1+w_{1}(t, y)}\right\}\right)
$$

whenever $t, y \in \mathfrak{\Im} \cap\left\{T_{\beta} S_{\sigma}\left(c_{n}\right)\right\},(t, y) \in L(Y), \sigma \in \Omega, \beta \in \Phi$, and $H_{w_{1}}\left(S_{\sigma} t, T_{\beta} y\right)>0$.

Assume that $\mathfrak{I}$ is regular and satisfies the $\Delta_{M}$-condition. Then, $\left\{T_{\beta} S_{\sigma}\left(c_{n}\right)\right\}$ is a sequence in $\mathfrak{J},\left(c_{n}, c_{n+1}\right) \in L(Y)$, and $\left\{T_{\beta} S_{\sigma}\left(c_{n}\right)\right\} \longrightarrow g^{*}$. Also, if $g^{*}$ satisfies (45) and $\left(c_{n}, g^{*}\right) \in L(Y)$ or $\left(g^{*}, c_{n}\right) \in L(Y)$ for each $n \in \mathbb{N} \cup\{0\}$, then $S_{\sigma}$ and $T_{\beta}$ have common fixed point $g^{*}$ in $\mathfrak{\Im}$ for all $\sigma \in \Omega$ and $\beta \in \Phi$.
Proof. Define $\alpha: \mathfrak{I} \times \mathfrak{I} \longrightarrow[0, \infty)$ by $\alpha(t, y)=1$ if $t \in \mathfrak{I}$ and $(t, y) \in L(Y)$. Otherwise, $\alpha(t, y)=0$. By the definition of the graph dominated on $\mathfrak{I}$, we have $(t, y) \in L(Y)$ for all $y \in S_{\sigma} t$ and $(t, y) \in L(Y)$ for each $y \in T_{\beta} t$. So, $\alpha(t, y)=1$ for all $y \in S_{\sigma} t$ and $\alpha(t, y)=1$ for every $y \in T_{\beta} t$. This means that $\inf \left\{\alpha(t, y): y \in S_{\sigma} t\right\}=1$ and $\inf \left\{\alpha(t, y): y \in T_{\beta} t\right\}=1$. Hence, $\alpha_{*}\left(t, S_{\sigma} t\right)=1$ and $\alpha_{*}\left(t, T_{\beta} t\right)=1$ for every $t \in \mathfrak{J}$. So, the families of mappings are $\alpha_{*}$-dominated on $\mathfrak{I}$. Furthermore, inequality (45) can be expressed as

$$
\tau+U\left(H_{w_{1}}\left(S_{\sigma} t, T_{\beta} y\right)\right) \leq U\left(\max \left\{w_{1}(t, y), w_{1}\left(t, S_{\sigma} t\right), \frac{w_{2}\left(t, T_{\beta} y\right)}{2}, \frac{w_{1}\left(t, S_{\sigma} t\right) \cdot w_{1}\left(y, T_{\beta} y\right)}{1+w_{1}(t, y)}\right\}\right)
$$

whenever $\quad t, y \in \mathfrak{I} \cap\left\{T_{\beta} S_{\sigma}\left(c_{n}\right)\right\}, \quad \alpha(t, y) \geq 1, \quad$ and $H_{w_{1}}\left(S_{\sigma} t, T_{\beta} y\right)>0$. Also, (ii) holds. Then, by Theorem 1, we have $\left\{T_{\beta} S_{\sigma}\left(c_{n}\right)\right\}$ is a sequence in $\mathfrak{J}$, and $\left\{T_{\beta} S_{\sigma}\left(c_{n}\right)\right\} \longrightarrow g^{*} \in \mathfrak{I}$. Now, $c_{n}, g^{*} \in \mathfrak{I}$, and either $\left(c_{n}, g^{*}\right) \in L(Y)$ or $\left(g^{*}, c_{n}\right) \in L(Y)$ implies that either $\alpha\left(c_{n}, g^{*}\right) \geq 1$ or $\alpha\left(g^{*}, c_{n}\right) \geq 1$. So, all the requirements of Theorem 1 are satisfied. Hence, from Theorem $1, S_{\sigma}$ and $T_{\beta}$ have a common fixed point $g^{*}$ in $\mathfrak{I}$, and $w_{1}\left(g^{*}, g^{*}\right)=0$.

\section{Results on Single-Valued Mappings}

In this section, some consequences of our results related to single-valued mappings in m.l.m. spaces have been discussed. Let $(\mathfrak{I}, w)$ be an m.l.m. space, $c_{0} \in \mathfrak{I}$, and $S_{\sigma}, T_{\beta}: \mathfrak{I} \longrightarrow \mathfrak{I}$ be two families of mappings. Let $c_{1}=S_{\sigma} c_{0}$, 
$c_{2}=T_{\beta} c_{1}$, and $c_{3}=S_{\sigma} c_{2}$. Adopting this way, we make a sequence $c_{n}$ in $\mathfrak{\Im}$ so that $c_{2 n+1}=S_{\sigma} c_{2 n}$ and $c_{2 n+2}=T_{\beta} c_{2 n+1}$, where $n=0,1,2, \ldots$. We represent this kind of iterative sequence by $\left\{T_{\beta} S_{\sigma}\left(c_{n}\right)\right\}$. We say that $\left\{T_{\beta} S_{\sigma}\left(c_{n}\right)\right\}$ is a sequence in $\Im$ generated by $c_{0}$. If $\left\{S_{\sigma}: \sigma \in \Omega\right\}=\left\{T_{\beta}: \beta \in \Phi\right\}$, then we denote $\left\{\Im S_{\sigma}\left(c_{n}\right)\right\}$ instead of $\left\{T_{\beta} S_{\sigma}\left(c_{n}\right)\right\}$.
Theorem 3. Let $(\mathfrak{I}, w)$ be a complete m.l.m. space. Assume that $w$ is regular and satisfies the $\triangle_{M}$-condition. Let $r>0$, $c_{0} \in \overline{B_{w_{1}}\left(c_{0}, r\right) \subseteq} \mathfrak{I}, \alpha: \mathfrak{I} \times \mathfrak{I} \longrightarrow[0, \infty)$, and $\left\{S_{\sigma}: \sigma \in \Omega\right\}$, $\left\{T_{\beta}: \beta \in \Phi\right\}$ be two families of $\alpha$-dominated mappings from $\Im$ to $\mathfrak{~}$. Then, there exist $\tau>0$ and $U \in \mathrm{F}$ such that

$$
\tau+U\left(H_{w_{1}}\left(S_{\sigma} t, T_{\beta} g\right)\right) \leq U\left(\max \left\{w_{1}(t, g), w_{1}\left(t, S_{\sigma} t\right), \frac{w_{2}\left(t, T_{\beta} g\right)}{2}, \frac{w_{1}\left(t, S_{\sigma} t\right) \cdot w_{1}\left(g, T_{\beta} g\right)}{1+w_{1}(t, g)}\right\}\right)
$$

whenever $t, g \in \Im \cap\left\{T_{\beta} S_{\sigma}\left(c_{n}\right)\right\}, \alpha(t, g) \geq 1, \sigma \in \Omega, \beta \in \Phi$, and $w_{1}\left(S_{\sigma} t, T_{\beta} g\right)>0$.

Then, $\left\{T_{\beta} S_{\sigma}\left(c_{n}\right)\right\}$ is a sequence in $\mathfrak{I}, \alpha\left(c_{n}, c_{n+1}\right) \geq 1$ for each $n \in \mathbb{N} \cup\{0\}$ and $\left\{T_{\beta} S_{\sigma}\left(c_{n}\right)\right\} \longrightarrow h \in \mathfrak{J}$. Also, if $u$ satisfies (47) and either $\alpha\left(c_{n}, h\right) \geq 1$ or $\alpha\left(h, c_{n}\right) \geq 1$ for all $n \in \mathbb{N} \cup\{0\}$, then $S_{\sigma}$ and $T_{\beta}$ have a common fixed point $h$ in $\mathfrak{J}$ for every $\sigma \in \Omega$ and $\beta \in \Phi$.

Proof. The proof is similar to the proof of Theorem 1.
If we take $\left\{S_{\sigma}: \sigma \in \Omega\right\}=\left\{T_{\beta}: \beta \in \Phi\right\}$ in Theorem 3, then we can get the following result.

Corollary 2. Let $(\mathfrak{I}, w)$ be a complete m.l.m. space. Assume that $w$ is regular and satisfies the $\Delta_{M}$-condition. Let $c_{0} \in \mathfrak{J}$, $\alpha: \mathfrak{I} \times \mathfrak{I} \longrightarrow[0, \infty)$, and $\left\{S_{\sigma}: \sigma \in \Omega\right\}$ be a family of $\alpha$-dominated mapping from $\mathfrak{I}$ to $\mathfrak{\Im}$. Then, there exist $\tau>0$ and $U \in \mathrm{F}$ such that

$$
\tau+U\left(H_{w_{1}}\left(S_{\sigma} t, S_{\beta} g\right)\right) \leq U\left(\max \left\{w_{1}(t, g), w_{1}\left(t, S_{\sigma} t\right), \frac{w_{2}\left(t, T_{\beta} g\right)}{2}, \frac{w_{1}\left(t, S_{\sigma} t\right) \cdot w_{1}\left(g, S_{\beta} g\right)}{1+w_{1}(t, g)}\right\}\right),
$$

whenever $t, g \in \mathfrak{I} \cap\left\{\Im S_{\sigma}\left(c_{n}\right)\right\}, \quad \alpha(t, g) \geq 1, \quad \sigma, \beta \in \Omega$, and $w_{1}\left(S_{\sigma} t, S_{\sigma} g\right)>0$. Then, $\left\{\mathfrak{I} S_{\sigma}\left(c_{n}\right)\right\}$ is a sequence in $\mathfrak{I}$, $\alpha\left(c_{n}, c_{n+1}\right) \geq 1 \quad$ for each $n \in \mathbb{N} \cup\{0\}$, and $\left\{\mathfrak{J} S_{\sigma}\left(c_{n}\right)\right\} \longrightarrow h \in \mathfrak{J}$. Also, if $h$ satisfies (48) and either $\alpha\left(c_{n}, h\right) \geq 1$ or $\alpha\left(h, c_{n}\right) \geq 1$ for each $n \in \mathbb{N} \cup\{0\}$, then $h$ is the fixed point of $S_{\sigma}$ in $\mathfrak{I}$ for every $\sigma \in \Omega$.

\section{Application in Integral Equations}

In this section, we discuss the application of our work in integral equations. First of all, we present our main result without a closed ball for self-mappings and without $\alpha_{*}$-dominated functions and then apply it to attain an application in integral equations. We also discuss the uniqueness.

Theorem 4. Let $(\mathfrak{I}, w)$ be a complete m.l.m. space. Assume that $w$ is regular and satisfies the $\Delta_{M}$-condition. Let $c_{0} \in \mathfrak{I}$ and $\left\{S_{\sigma}: \sigma \in \Omega\right\}$ and $\left\{T_{\beta}: \beta \in \Phi\right\}$ be the families of selfmappings. Then, there exist $\tau>0$ and a strictly increasing function $U: \mathbb{R}_{+} \longrightarrow \mathbb{R}$ such that

$$
\tau+U\left(H_{w_{1}}\left(S_{\sigma} t, T_{\beta} g\right)\right) \leq U\left(\max \left\{w_{1}(t, g), w_{1}\left(t, S_{\sigma} t\right), \frac{w_{2}\left(t, T_{\beta} g\right)}{2}, \frac{w_{1}\left(t, S_{\sigma} t\right) \cdot w_{1}\left(g, T_{\beta} g\right)}{1+w_{1}(t, g)}\right\}\right)
$$

whenever $t, g \in\left\{T_{\beta} S_{\sigma}\left(c_{n}\right)\right\}, \quad \sigma \in \Omega, \quad \beta \in \Phi, \quad$ and $w_{1}\left(S_{\sigma} t, T_{\beta} g\right)>0$. Then, $\left\{T_{\beta} S_{\sigma}\left(c_{n}\right)\right\} \longrightarrow h \in \mathfrak{J}$. Also, if inequality (49) holds for $t, g \in\{h\}$, then $S_{\sigma}$ and $T_{\beta}$ have a unique common fixed point $h$ in $\mathfrak{\Im}$ for all $\sigma \in \Omega$ and $\beta \in \Phi$.
Proof. The proof is similar to the proof of Theorem 1. We prove only uniqueness. Let $S_{\sigma}$ and $T_{\beta}$ have another common fixed point $v$. Suppose $w_{1}\left(S_{\sigma} u, T_{\beta} v\right)>0$. Then,

$$
\tau+U\left(w_{1}\left(S_{\sigma} u, T_{\beta} v\right)\right) \leq U\left(\max \left\{w_{1}(u, v), w_{1}\left(u, S_{\sigma} u\right), \frac{w_{2}\left(u, T_{\beta} v\right)}{2}, \frac{w_{1}\left(u, S_{\sigma} u\right) \cdot w_{1}\left(v, T_{\beta} v\right)}{1+w_{1}(u, v)}\right\}\right) .
$$


This implies that

$$
w_{1}(u, v)<\mu_{1} w_{1}(u, v)<w_{1}(u, v),
$$

which is not true. So, $w_{1}\left(S_{\sigma} u, T_{\beta} v\right)=0$. Hence, $u=v$.

Let $W=C\left([0,1], \mathbb{R}_{+}\right)$be the set of all continuous functions on $[0,1]$. Consider the families of integral equations

$$
\begin{gathered}
u(k)=\int_{0}^{k} H_{\sigma}(k, h, u(h)) \mathrm{d} h+\epsilon, \\
c(k)=\int_{0}^{k} G_{\beta}(k, h, c(h)) \mathrm{d} h+\epsilon,
\end{gathered}
$$

for all $k \in[0,1], \sigma \in \Omega, \beta \in \Phi$, and $H_{\sigma}, G_{\beta}$ are the functions from $[0,1] \times[0,1] \times W$ to $\mathbb{R}$. For $c \in C\left([0,1], \mathbb{R}_{+}\right)$, define the supremum norm as $\|c\|_{\tau}=\sup _{k \in[0,1]}\left\{|c(k)| e^{-\eta k}\right\}$, where $\eta>0$ is arbitrarily taken. Define

$$
w_{1}(c, p)=\frac{1}{2} \sup _{k \in[0,1]}\left\{|c(k)+p(k)| e^{-\tau k}\right\}=\frac{1}{2}\|c+p\|_{\tau},
$$

for all $\quad c, p \in C\left([0,1], \mathbb{R}_{+}\right)$; with these settings, $\left(C\left([0,1], \mathbb{R}_{+}\right), d_{\tau}\right)$ becomes a complete m.l.m. space.

Now, we prove the following theorem to ensure the uniqueness and existence of a solution of families of nonlinear integral equations (52) and (53).

Theorem 5. Assume the following constraints are satisfied:

(i) $\left\{H_{\sigma}, \sigma \in \Omega\right\}$ and $\left\{G_{\beta}, \beta \in \Phi\right\}$ are two families of mappings from $[0,1] \times[0,1] \times C\left([0,1], \mathbb{R}_{+}\right)$to $\mathbb{R}$. (ii) Define

$$
\begin{aligned}
& \left(S_{\sigma} u\right)(k)=\int_{0}^{k} H_{\sigma}(k, h, u(h)) \mathrm{d} h+\epsilon, \\
& \left(T_{\beta} c\right)(k)=\int_{0}^{k} G_{\beta}(k, h, c(h)) \mathrm{d} h+\epsilon .
\end{aligned}
$$

Suppose there exists $\tau>0$ such that

$$
\left|H_{\sigma}(k, h, u)+G_{\beta}(k, h, c)\right| \leq \frac{\tau E_{(\sigma, \beta)}(u, c)}{\tau E_{(\sigma, \beta)}(u, c)+1},
$$

for all $k, h \in[0,1]$ and $u, c \in C\left([0,1], \mathbb{R}^{+}\right)$, where

$$
E_{(\sigma, \beta)}(u, c)=\max \left(\frac{1}{2}\left\{\begin{array}{c}
\|u+c\|_{\tau},\left\|u+S_{\sigma} u\right\|_{\tau^{\prime}} \\
\frac{\left\|u+S_{\sigma} u\right\|_{\tau}+\left\|c+T_{\beta} c\right\|_{\tau}}{2}, \\
\frac{\left\|u+S_{\sigma} u\right\|_{\tau^{*}}\left\|c+T_{\beta} c\right\|_{\tau}}{1+\|u+c\|_{\tau}}
\end{array}\right\}\right)
$$

Then, integral equations (52) and (53) have a unique solution.

Proof. By assumption (ii),

$$
\begin{aligned}
\left|S_{\sigma} u+T_{\beta} c\right| & =\int_{0}^{k}\left|H_{\sigma}(k, h, u)+G_{\beta}(k, h, c)\right| \mathrm{d} h \leq \int_{0}^{k} \frac{\tau E_{(\sigma, \beta)}(u, c)}{\tau E_{(\sigma, \beta)}(u, c)+1} e^{\tau h} \mathrm{~d} h \\
& \leq \frac{\tau E_{(\sigma, \beta)}(u, c)}{\tau E_{(\sigma, \beta)}(u, c)+1} \int_{0}^{k} e^{\tau h} \mathrm{~d} h \leq \frac{E_{(\sigma, \beta)}(u, c)}{\tau E_{(\sigma, \beta)}(u, c)+1} e^{\tau k} .
\end{aligned}
$$

This implies

$$
\begin{aligned}
& \left|S_{\sigma} u+T_{\beta} c\right| e^{-\tau k} \leq \frac{E_{(\sigma, \beta)}(u, c)}{\tau E_{(\sigma, \beta)}(u, c)+1}\left\|S_{\sigma} u+T_{\beta} c\right\|_{\tau} \leq \frac{E_{(\sigma, \beta)}(u, c)}{\tau E_{(\sigma, \beta)}(u, c)+1} \\
& \frac{\tau E_{(\sigma, \beta)}(u, c)+1}{E_{(\sigma, \beta)}(u, c)} \leq \frac{1}{\left\|S_{\sigma} u+T_{\beta} c\right\|_{\tau}} \tau+\frac{1}{E_{(\sigma, \beta)}(u, c)} \leq \frac{1}{\left\|S_{\sigma} u+T_{\beta} c\right\|_{\tau}},
\end{aligned}
$$

which further implies

$$
\tau-\frac{1}{\left\|S_{\sigma} u(k)+T_{\beta} c(k)\right\|_{\tau}} \leq \frac{-1}{E_{(\sigma, \beta)}(u, c)} .
$$

So, all the requirements of Theorem 5 are satisfied for $U(f)=-1 / \sqrt{f} ; f>0$ and $w_{1}(f, c)=1 / 2\|f+c\|_{\tau}$. Hence, two families of integral equations given in (52) and (53) have a unique common solution.

\section{Conclusion}

In this article, we have achieved some new results for setvalued mappings belonging to two families which satisfy 
generalized rational-type Wardowski's contraction. Dominated mappings are applied to find out the fixed point results. Applications in the subject of integral equations and graph theory are presented. Moreover, we investigate our results in new generalized modular-like metric spaces. Many consequences of our results in dislocated metric spaces, metric spaces, and partial metric spaces (even with a partial order) can be established easily.

\section{Data Availability}

The data used to support the findings of this study are available from the corresponding author upon request.

\section{Conflicts of Interest}

The authors declare that they have no conflicts of interest.

\section{Authors' Contributions}

Each author contributed equally to this paper, read, and approved the final manuscript.

\section{Acknowledgments}

The authors thank the Basque Government for supporting this work through Grant IT1207-19.

\section{References}

[1] Ö. Acar, G. Durmaz, and G. Minak, "Generalized multivalued F-contractions on complete metric spaces," Bulletin of the Iranian Mathematical Society, vol. 40, pp. 1469-1478, 2014.

[2] N. Alamgir, Q. Kiran, H. Işık, and H. Aydi, "Fixed point results via a Hausdorff controlled type metric," Advances in Difference Equations, vol. 2020, p. 24, 2020.

[3] E. Ameer, H. Aydi, M. Arshad, and M. De la Sen, "Hybrid ćirić type graphic $(\Upsilon, \Lambda)$-contraction mappings with applications to electric circuit and fractional differential equations," Symmetry, vol. 12, no. 3, p. 467, 2020.

[4] H. Aydi, H. Lakzian, Z. D. Mitrović, and S. Radenović, "Best proximity points of MT-cyclic contractions with property UC," Numerical Functional Analysis and Optimization, vol. 41, no. 7, pp. 871-882, 2020.

[5] S. Banach, "Sur les opérations dans les ensembles abstraits et leur application aux équations intégrales," Fundamenta Mathematicae, vol. 3, pp. 133-181, 1922.

[6] P. Chaipunya, Y. Je Cho, and P. Kumam, "Geraghty-type theorems in modular metric spaces with an application to partial differential equation," Advances in Difference Equations, vol. 2012, no. 1, p. 83, 2012.

[7] V. V. Chistyakov, "Modular metric spaces, I: basic concepts," Nonlinear Analysis: Theory, Methods \& Applications, vol. 72, no. 1, pp. 1-14, 2010.

[8] N. Hussain, J. Ahmad, and A. Azam, "On Suzuki-Wardowski type fixed point theorems," Journal of Nonlinear Sciences and Applications, vol. 8, no. 6, pp. 1095-1111, 2015.

[9] N. Hussain, S. Al-Mezel, and P. Salimi, "Fixed points for $\psi$-graphic contractions with application to integral equations," Abstract and Applied Analysis, vol. 2013, Article ID 575869, 11 pages, 2013.
[10] N. Hussain and P. Salimi Salimi, "Suzuki-Wardowski type fixed point theorems for $\alpha$-gf-contractions," Taiwanese Journal of Mathematics, vol. 18, no. 6, pp. 1879-1895, 2014.

[11] J. Jachymski, "The contraction principle for mappings on a metric space with a graph," Proceedings of the American Mathematical Society, vol. 4, no. 136, pp. 1359-1373, 2008.

[12] D. Jain, A. Padcharoen, P. Kumam, and D. Gopal, "A new approach to study fixed point of multivalued mappings in modular metric spaces and applications," Mathematics, vol. 4, no. 3 , p. 51, 2016.

[13] K. Kuaket and P. Kumam, "Fixed points of asymptotic pointwise contractions in modular spaces," Applied Mathematics Letters, vol. 24, no. 11, pp. 1795-1798, 2011.

[14] P. Kumam, "Fixed point theorems for nonexpansive mapping in modular spaces," Archiv der Mathematik, vol. 40, pp. 345-353, 2004.

[15] S. B. Nadler, "Multi-valued contraction mappings," Pacific Journal of Mathematics, vol. 30, no. 2, pp. 475-488, 1969.

[16] A. Padcharoen, D. Gopal, P. Chaipunya, and P. Kumam, "Fixed point and periodic point results for $\alpha$-type $F$-contractions in modular metric spaces," Fixed Point Theory and Applications, vol. 2016, p. 39, 2016.

[17] H. Piri and P. Kumam, "Some fixed point theorems concerning F-contraction in complete metric spaces," Fixed Point Theory and Applications, vol. 2014, p. 210, 2014.

[18] H. Piri, S. Rahrovi, H. Marasi, and P. Kumam, "A fixed point theorem for F-Khan-contractions on complete metric spaces and application to integral equations," The Journal of Nonlinear Sciences and Applications, vol. 10, no. 9, pp. 4564-4573, 2017.

[19] V. Parvaneh, M. R. Haddadi, and H. Aydi, "On best proximity point results for some type of mappings," Journal of Function Spaces, vol. 2020, Article ID 6298138, 6 pages, 2020.

[20] Q. Mahmood, A. Shoaib, T. Rasham, and A. Muhammad, "Fixed point results for the family of mulțivalued F-contractive mappings on closed ball in complete dislocated b-metric spaces," Mathematics, vol. 7, no. 1, 2019.

[21] T. Rasham, A. Shoaib, B. S. Alamri, and M. Arshad, "Multivalued fixed point results for new generalized $F$-dominted contractive mappings on dislocated metric space with application," Journal of Function Spaces, vol. 2018, Article ID 4808764, 12 pages, 2018.

[22] T. Rasham, A. Shoaib, N. Hussain, M. Arshad, and S. U. Khan, "Common fixed point results for new ciric-type rational multivalued F-contraction with an application," Journal of Fixed Point Theory and Applications, vol. 20, no. 1, p. 45, 2018.

[23] T. Rasham and A. Shoaib, "Common fixed point results for two families of multivalued $A$-dominated contractive mappings on closed ball with applications," Open Mathematics, vol. 17, no. 1, pp. 1350-1360, 2019.

[24] T. Rasham, A. Shoaib, G. Marino, B. A. S. Alamri, and M. Arshad, "Sufficient conditions to solve two systems of integral equations via fixed point results," Journal of Inequalities and Applications, vol. 1, p. 182, 2019.

[25] T. Rasham, A. Shoaib, N. Hussain, B. A. S. Alamri, and M. Arshad, "Multivalued fixed point results in dislocated $b$ metric spaces with application to the system of nonlinear integral equations," Symmetry, vol. 11, no. 1, p. 40, 2019.

[26] T. Rasham, Q. Mahmood, A. Shahzad, A. Shoaib, and A. Azam, "Some fixed point results for two families of fuzzy Adominated contractive mappings on closed ball," Journal of Intelligent \& Fuzzy Systems, vol. 36, no. 4, pp. 3413-3422, 2019. 
[27] T. Rasham, G. Marino, and A. Shoaib, "Fixed points for a pair of $F$-dominated contractive mappings in rectangular $b$-metric spaces with graph," Mathematics, vol. 7, no. 10, p. 884, 2019.

[28] T. Rasham, A. Shoaib, Q. Zaman, and M. S. Shabbir, "Fixed point results for a generalized $F$-contractive mapping on closed ball with application," Mathematical Sciences, vol. 14, no. 2, pp. 177-184, 2020.

[29] M. Sgroi and C. Vetro, "Multi-valued F-contractions and the solutions of certain functional and integral equations," Filomat, vol. 27, no. 7, pp. 1259-1268, 2013.

[30] W. Shatanawi, E. Karapinar, H. Aydi, and A. Fulga, "Wardowski type contractions with applications on Caputo type nonlinear fractional differential equations," Journal of Applied Mathematics and Physics, vol. 82, no. 2, pp. 157-170, 2020.

[31] A. Shazad, T. Rasham, G. Marino, and A. Shoaib, "On fixed point results for $\alpha_{*}-\psi$-dominated fuzzy contractive mappings with graph," Journal of Intelligent \& Fuzzy Systems, vol. 38, no. 8, pp. 3093-3103, 2020.

[32] A. Shoaib, "Fixed point results for $\alpha_{*}-\psi$-mulțivalued mappings," Bulletin of Mathematical Analysis and Applications, vol. 8, no. 4, pp. 43-55, 2016.

[33] A. Shoaib, A. Hussain, M. Arshad, and A. Azam, "Fixed point results for $\alpha_{*}-\psi$-ciric type multivalued mappings on an intersection of a closed ball and a sequence with graph," Journal of Mathematical Analysis and Applications, vol. 7, no. 3, pp. 41-50, 2016.

[34] S. S. S. Radenović and C. Vetro, "Graphical metric space: a generalized setting in fixed point theory," Revista de la Real Academia de Ciencias Exactas, Fisicas y Naturales. Serie A. Matematicas, vol. 111, no. 3, pp. 641-655, 2017.

[35] C. Vetro and F. Vetro, "Metric or partial metric spaces endowed with a finite number of graphs: a tool to obtain fixed point results," Topology and Its Applications, vol. 164, pp. 125-137, 2014.

[36] C. Vetro and D. Wardowski, "Krasnoselskii-Schaefer type method in the existence problems," Topological Methods in Nonlinear Analysis, vol. 54, no. 1, pp. 131-139, 2019.

[37] F. Vetro, "Fixed point for $\alpha-\Theta-\Phi$-contractions and first-order periodic differential problem," Revista de la Real Academia de Ciencias Exactas, Físicas y Naturales. Serie A. Matemáticas, vol. 113, no. 3, pp. 1823-1837, 2019.

[38] F. Vetro, " $\mu$-contractions in ordered metric spaces endowed with a $\omega_{0}$-distance," Filomat, vol. 32 , no. 10, pp. 3725-3730, 2018.

[39] D. Wardowski, "Fixed point theory of a new type of contractive mappings in complete metric spaces," Fixed Point Theory and Applications, vol. 2012, p. 94, 2012.

[40] M. Abbas, D. Gopal, D. K. Patel, and C. Vetro, "Some coincidence and periodic points results in a metric space endowed with a graph and applications," Banach Journal of Mathematical Analysis, vol. 9, no. 3, pp. 128-139, 2015. 